\title{
A longitudinal study of risk factors for motorcycle crashes among junior college students in Taiwan
}

\author{
Mau-Roung Lin ${ }^{\mathrm{a}, *}$, Shu-Hui Chang ${ }^{\mathrm{b}}$, Lu Pai ${ }^{\mathrm{c}}$, Penelope M. Keyl ${ }^{\mathrm{d}}$ \\ a Institute of Injury Prevention and Control, Taipei Medical University, 250 Wu-Hsing Street, Taipei 110, Taiwan, ROC \\ ${ }^{\mathrm{b}}$ Department of Public Health, School of Public Health, National Taiwan University, 1 Jen-Ai Road, Section 1, Taipei 100, Taiwan, ROC \\ ${ }^{\mathrm{c}}$ School of Public Health, National Defense Medical Center, 161 Minchuan East Road, Section 6, Taipei 114, Taiwan, ROC \\ d Johns Hopkins Department of Emergency Medicine, 1830 East Monument Street, Suite 6-100, Baltimore, MD 21205, USA
}

Received 15 May 2001; received in revised form 9 November 2001; accepted 14 December 2001

\begin{abstract}
A cohort of 4729 junior college students in an urban and a rural area in Taiwan was followed up for a period of 20 months. Students' characteristics, including riding exposures, as well as human, vehicular, and environmental factors were collected using one initial and three follow-up questionnaires. The Anderson-Gill (AG) multiplicative intensity model was used to determine the risk of a motorcycle crash over time while also allowing for the modeling of multiple events. The average response rate for the four assessments was $92 \%$. The adjusted relative hazard (RH) for students living in the rural as opposed to the urban area for crashes was 1.67 at the beginning of the study but decreased to 0.66 by the end. Past motorcycle crash history, number of riding days, average riding distance, risk-taking level, alcohol consumption, and traffic violations were all significantly associated with an increased risk of being involved in a crash. Conversely, increasing age, riding experience, and automobile licensure were related to a decreased risk of crashing. Furthermore, helmet use was not independently related to the risk of crashing. In conclusion, a high-risk group predisposed to involvement in a motorcycle crash, including both non-injury and injury-related crashes, can be identified using selected risk factors for crash prevention among young riders. (C) 2002 Elsevier Science Ltd. All rights reserved.
\end{abstract}

Keywords: Adolescents; Motorcycle crashes; Motorcycle injuries; Risk factors; Taiwan

\section{Introduction}

Risk factors for motorcycle injuries, particularly for severe injuries, have been widely studied (Baker and Fisher, 1977; Begg et al., 1994; Braddock et al., 1992; Kraus et al., 1975b; Krishnan and Smith, 1994). However, previous studies have not included motorcycle crashes involving minor injuries and those not involving injuries.

It is important to determine the risk factors for all motorcycle crashes based on the following considerations. First, although severe motorcycle injuries result in tremendous medical costs (Bach and Wyman, 1986; Bray et al., 1985), the largest economic cost of motor-vehicle crashes results from property-damage-only and minor-injury crashes (those with a score on the Abbreviated Injury Scale (AIS) of 0 or 1) (Blincose, 1994). Such crashes accounted for $51 \%$ of the economic costs of motor-vehicle crashes in the US in 1994 (Blincose, 1994). Second, the prevention of collisions

\footnotetext{
* Corresponding author. Tel.: +886-2-27390217; fax: +886-2-27390387.

E-mail address: mrlin@tmu.edu.tw (M.-R. Lin).
}

involving property-damage-only and minor injuries can significantly improve the quality of life for individuals, since minor injuries account for $88 \%$ of quality-adjusted life years lost due to injury in the first 6 months after injury and for 91\% over the remaining lifetime (McClure and Douglas, 1996). Third, police and hospital records are traditionally utilized by motorcycle-injury studies, but these data usually over-represent severely injured riders (Rutledge and Stutts, 1993). Hence, a representative sample of motorcycle crashes is required to obtain an unbiased result as well as to validate those results from police and hospital records. Finally, since opponents of helmet laws allege that helmets increase rider fatigue, which can then cause more crashes (American Medical Association, 1977), the relationship of helmet use to the risk of crashing needs to be empirically examined.

Like in many Asian countries, motorcycles in Taiwan are one of the most important forms of transportation. According to police reports, $43 \%$ of the 2717 deaths from traffic injuries were motorcycle riders in 1992, and of these motorcyclist deaths, $31 \%$ were young riders aged 16-25 years (Ministry of Communication and Transportation, 
1993). Furthermore, emergency room records have shown that more than $66 \%$ of motorcycle injuries occur to young riders aged less than 20 years (Chao and Tan, 1984; Ding et al., 1993; Wu et al., 1992). Therefore, it is appropriate to construct a cohort study of Taiwan's adolescents due to the high occurrence of motorcycle injuries in order to collect a representative sample of motorcycle crashes. This cohort design of motorcycle injuries might not be applicable to the situation in many Western countries.

This 20 month longitudinal cohort study of junior college students from an urban and a rural area of Taiwan sought to determine the risk factors for motorcycle crashes.

\section{Materials and methods}

\subsection{Study population and design}

Junior college students from two residential areas, Taipei (an urbanized area in northern Taiwan with a population density of 10,001 persons per square kilometer) and Hualien (a rural area on the central east coast with a population density of 76 persons per square kilometer), were selected for this study. Two junior colleges from each area were randomly selected from a total of eight junior colleges in Taipei and three in Hualien. Because one of the selected colleges in Taipei declined to participate, only three junior colleges were included in the study.

Students attending junior colleges in Taiwan complete a series of five grades before graduation with the first three grades corresponding to the 3 years of high school. Students in the first and second years, who have fewer opportunities to ride a motorcycle, were not included in this study. Furthermore, students in Taipei who were already in their fifth year at the beginning of the study were excluded because the study was initiated 2 months later in Taipei than in Hualien, and fifth-year students would have only contributed 2-4 months of follow-up data to the study. The study population initially consisted of 4721 students (2224 from Taipei and 2497 from Hualien) who were enrolled at the three participating schools at the time of the initial assessment.

One initial and three follow-up assessments were conducted at the beginning of the study and at about 6 month intervals, respectively. All assessments used self-administered structured questionnaires developed by focus group discussions and a pretest. To accommodate the school schedule, the initial assessment was begun in November 1994 in Hualien, and in February 1995 in Taipei. Motorcycle crashes occurring in the study period were principally reported through student representatives on an ongoing basis. Nonetheless, a small number of crashes was only ascertained by student reports at the follow-up assessments and from school records.

This research was reviewed and approved by the Institutional Review Board at the National Defense Medical
Center in Taiwan, and informed consent was obtained from all participants and the authorities of the three schools.

\subsection{Exposure assessment}

Motorcycles were defined as all types of two-wheeled motorized vehicles including scooters and single and multiple-stroke motorcycles. At each assessment, motorcycle riders were defined as students who rode a motorcycle as an operator or a passenger on a public road, at least once in the month prior to the assessment.

Permanent characteristics were only measured during the initial assessment. In the four assessments, if students rode a motorcycle at least once in the month prior to assessment, their other time-dependent characteristics would continue to be measured. In order to organize the variables of the study, potential risk factors were categorized into four general areas: riding exposure, and human, vehicular, and environmental factors. Permanent characteristics in the study included: (1) human factors: age at initial assessment, gender, weight, height, socioeconomic status based on parental education and income, whether the student lived at home, motorcycle crash history, history of medical treatment due to a motorcycle crash, health problems (such as myopia, astigmatisms, and chronic diseases or physical handicaps), and risk-taking level as measured by 14 items (i.e. video game playing at stores, cigarette smoking, jaywalking, skipping class, motorcycle/car racing on roads, chewing betel nut, drinking and driving, cheating on examinations, using foul language, going to pubs/bars, verbal fighting, sexual behavior, physical fighting, and gambling). These items were identified from two focus group sessions (one was held among six health-related professionals and the other among six junior students) and one pretest with 94 junior college students, and more details are described elsewhere (Lin et al., 2002) (2) environmental factors: residential area (rural or urban). Time-dependent characteristics included: (1) riding exposure: riding a motorcycle at least once during the last month, days of motorcycle use during the last 2 weeks, and average riding time and average riding distance per riding day during the last 2 weeks; (2) human factors: riding experience, motorcycle and automobile licensure, helmet ownership, frequency of helmet use during the last 2 weeks, alcohol consumption during the last month, usual riding speed, the ownership of the motorcycle frequently used, and presence of registered traffic violations during the last 6 months; (3) vehicular factors: make, model year, and engine volume of the motorcycle frequently used; (4) environmental factors: frequently used road type.

\subsection{Outcome assessment}

A motorcycle crash was defined as having occurred when the rider(s) fell down onto the road either in a single or multiple-vehicle collision. The information about crashes was mainly collected through 101 class representatives 
chosen by their classmates, and each representative covered 23-60 students, depending on the size of his/her class. Students were asked to report any crashes occurring in the past week to their representatives at a weekly class meeting. When a crash was reported, the representative asked the student to complete a structured motorcycle crash questionnaire to provide information on the date and other details about the crash.

Several methods were used to facilitate representatives and students' participation in the study. First, at the beginning of the study, we explained to students that injuries from motorcycle crashes among students are a serious public health problem that they might help to reduce by providing information about their crash-related experiences over the study period. Second, an economic incentive was also provided to the representatives during the study period. Third, researchers also contacted the representatives by telephone every 2 weeks to remind them to report any motorcycle crashes that had occurred in the previous 2 weeks.

At each follow-up assessment visit, students who had been involved in a crash they had not reported to representatives were asked to complete the motorcycle crash questionnaire at that time. School records were also checked monthly to examine whether some severe crashes had not been reported, since it is compulsory for schools in Taiwan to record severe injuries, such as injuries requiring hospitalization and fatalities. When a recorded injury involving motorcycle use had not been reported by the student representative or the victim, researchers interviewed the victim by telephone. When the student had died from the crash, school and hospital records and his/her family or a close classmate were interviewed in person to obtain the corresponding crash information.

\subsection{Statistical methods}

Factor analysis was carried out on the 14 items used to construct risk-taking behavior. All items were found to load on one factor. Factor scores were computed using factor loadings and then categorized into tertiles. Both the identification of only a single factor and the factor loadings were reliable when factor analysis was carried out on two subsets created by randomly splitting the study population.

The relationship of the risk of a motorcycle crash to the potential risk factors was investigated using the Anderson-Gill (AG) multiplicative intensity model (Andersen and Gill, 1982), which is a generalization of the Cox proportional hazard model. In the AG model, the intensity function at a time point, which is an instantaneous rate of occurrence of a motorcycle crash at a time point for a student, is assumed to be multiplicatively related to the risk factors. Furthermore, the number of motorcycle crashes prior to this time point related to the past history of crashes is included explicitly in the model as a covariate to capture the dependency among multiple crashes for a student (Cook and Lawless, 1997; Wei and Glidden, 1997). Thus, the AG model for $\lambda_{i}\left(t ; H_{i}(t)\right.$ ) (Fleming and Harrington, $1991)$, the instantaneous probability of occurrence of motorcycle crashes conditional on the student's past history of motorcycle crashes at time $t$ for student $i$, is expressed as

$\lambda_{i}\left(t ; H_{i}(t)\right)=\lambda_{0}(t) \exp \left(\beta^{\prime} Z_{i}(t)+\gamma X_{i}(t)\right)$,

where $t$ refers to the calendar time; $i=1, \ldots, n$ refers to students; $\lambda_{0}(t)$ is an arbitrary positive-valued function of time $t ; \beta^{\prime}$ is a vector of unknown regression parameters; $H_{i}(t)$ represents the history of motorcycle crashes up to time $t ; Z_{i}(t)$ is the vector of the covariate processes up to time $t ; \gamma$ is the regression parameter of $X_{i}(t)$; and $X_{i}(t)$ is the number of crashes prior to time $t$ representing a specified function of the history of motorcycle crashes. Since the process of motorcycle crashes in the AG model is measured by calendar time instead of the time from study entry, the delay of entry in the study of Taipei students can be adjusted spontaneously by using the calendar time scale in the AG model, and also seasonal effects due to different assessment time points between Taipei and Hualien are spontaneously removed. In addition, the time-dependent covariates in our study were measured at only four different time points, but not at each calendar time of occurrence of a crash. In the analysis, a direct method used the closest preceding values to impute the unmeasured time-dependent covariates at any given calendar time of a crash. SAS release 6.12 for PCs was used for all statistical analyses.

\section{Results}

\subsection{Response rates}

Based on the initial cohort of 4721 students enrolled during the semester of the initial assessment and taking into account that fifth-year students in Hualien were no longer followed-up after the second assessment because of graduation between the second and third assessments, response rates for the four assessments were 97, 95, 94, and 92\% in Taipei, and 93, 90, 89, and 88\% in Hualien.

Exposure rates of riding a motorcycle at least once during the previous month for the four assessments were 82, 85,91 , and $90 \%$, respectively. Only 90 students did not ride a motorcycle at least once in the previous month at all assessments they attended.

\subsection{Crash outcomes}

During the study period, 1150 motorcycle crashes occurred among Taipei students and 739 among Hualien students. Of students who completed at least one of the four assessments, 877 students (18.5\%) crashed once, $280(5.9 \%)$ twice, and $127(2.8 \%)$ at least three times. The detailed distribution of students by number of crashes is shown in Table 1. 
Table 1

Frequency distribution of motorcycle crashes by residential area occurring for each student over the 20 month study period

\begin{tabular}{|c|c|c|c|}
\hline Number of crashes & Taipei $n=2249$ frequency $(\%)$ & Hualien $n=2480$ frequency $(\%)$ & Total $n=4729$ frequency $(\%)$ \\
\hline 0 & $1498(66.6)$ & $1947(78.5)$ & $3445(72.8)$ \\
\hline 1 & 492 (21.9) & $385(15.5)$ & $877(18.5)$ \\
\hline 2 & $171(7.6)$ & $109(4.4)$ & $280(5.9)$ \\
\hline 3 & $56(2.5)$ & $28(1.1)$ & $84(1.8)$ \\
\hline 4 & $21(0.9)$ & $6(0.2)$ & $27(0.6)$ \\
\hline 5 & $5(0.2)$ & $3(0.1)$ & $8(0.2)$ \\
\hline 6 & $3(0.1)$ & $1(<0.1)$ & $4(0.1)$ \\
\hline 7 & $3(0.1)$ & $1(<0.1)$ & $4(0.1)$ \\
\hline
\end{tabular}

The incidence rates of crashes per 1000 person years were 427 in Taipei, 272 in Hualien, and 358 in the combined student cohort.

\subsection{Crude effects of permanent characteristics}

Table 2 shows the crude relative hazards (RHs) of the permanent characteristics for the occurrence of motorcycle crashes from univariate analysis in the AG model. By definition, the crude RH of students in a category of a characteristic as a reference group is 1.00 . The frequency and distribution of these factors measured at the initial assessment are also provided in this table. Without controlling for other characteristics, Hualien students were less likely than Taipei students to be involved in a motorcycle crash during the study period. Increased age at initial assessment was associated with a decreased risk of a motorcycle crash. Males and increased weight and height were associated with a greater risk of crashing. Students whose mothers had a high school education had a higher risk of crashing than did those whose mothers had an elementary school or no formal education.

Students affected by astigmatism or any chronic disease or physical handicap had a higher risk of motorcycle crashes than did those without. Students who had been involved in a motorcycle crash prior to the study were at a higher risk of experiencing a crash during the study period.

Students living at home were more likely to experience a crash than those who were not. Students in the middle or highest tertile for risk-taking level were at increased risk of crashing compared to those in the lowest tertile.

\subsection{Crude effects of time-dependent characteristics}

Table 3 shows the crude RHs of time-dependent characteristics for the occurrence of motorcycle crashes from univariate analysis in the AG model. The risk of motorcycle crashes was higher for students who operated a motorcycle as compared to those who were only passengers or those who did not ride during the last month. The risk of a motorcycle crash increased with an increase in the number of riding days, average riding time, and average riding distance per riding day. Furthermore, all three of these riding exposures showed a statistically significant linear association with risk of motorcycle crashes.

In the crude analysis, each previous motorcycle crash occurring in a study period was associated with an increased risk of a crash. Operators with more experience and automobile licenses were less likely to experience a crash. Riders who owned a partial or full-coverage helmet had a higher risk of crashing than those who did not own a helmet. Furthermore, there was a positive association between the risk of crashing and the number of days of helmet use.

A higher usual riding speed was positively associated with the risk of crashing. Riders who owned a motorcycle, drank alcohol, and had traffic violations had an increased risk of being involved in a motorcycle crash. Students who usually rode Yamaha motorcycles were at a somewhat higher risk than those riding other makes of motorcycles. Motorcycles made in 1995-1996 were associated with a greater risk of crashing than those made prior to 1994.

\subsection{Adjusted effects of permanent and time-dependent characteristics}

The results of multivariate analysis of data on potential risk factors are shown in Table 4. The adjusted RH of a crash for riders living in Hualien (rural area) compared to Taipei (urban area) at the time of initial assessment was 1.67. To take into account the changing effect of residential areas over time, an interaction between residential area and calendar time was considered in the model. Accordingly, the estimated RH values of Hualien to Taipei at 5, 10, and 17 months of follow-up were $1.23,0.90$, and 0.66 , respectively.

Each previous crash occurring during the study was associated with an increased risk of a subsequent crash $(\mathrm{RH}=$ 1.61). Having experienced a crash prior to the study also increased the risk of a crash during the study period $(\mathrm{RH}=$ 1.61). However, having experienced a crash prior to the study reduced the effect produced by the number of previous crashes occurring during the study period by a factor of 0.73 . For example, compared to students with no crash history, the RHs of those who had two previous crashes in the study period and also had at least a crash prior to the study and who had two previous crashes in the study period but had no 
Table 2

Crude relative hazards of time-independent characteristics for the occurrence of motorcycle crashes from the Anderson-Gill model analysis ${ }^{\mathrm{a}}$

Characteristic
Residential area
Taipei

Hualien

Age at initial assessment (year)

17

18

19

20

$\geq 21$

Sex

Female

Male

Body weight (kg)

Body height $(\mathrm{cm})$

Father's education

Elementary or below

High school

College or above

Mother's education

Elementary or below

High school

College or above

Parents' monthly income $(\mathrm{NT} \$)^{\mathrm{c}}$

$\leq 29999$ ( $\leq$ US\$ 1154)

30000-59999 (US\$ 1154-2308)

60000-89999 (US\$ 2308-3462)

90000-149999 (US\$ 3462-5769)

$\geq 150000$ (US\$ $\geq 5769$ )

Myopia

No

Yes

Astigmatism

No

Yes

Chronic disease or physical handicap

No

Yes

MTC crashes prior to this study

No

Yes

Received medical treatment for a MTC crash

No

Yes

Living at home

Yes

No

Risk-taking level

Lowest tertile

Middle tertile

Highest tertile
Frequency $(\%)$ at initial assessment

Relative hazard ${ }^{\mathrm{b}}(95 \% \mathrm{CI})$

$2154(48.3)$

$2308(51.7)$

1.00

$0.84(0.76-0.93)$

485 (10.9)

$1782(39.9)$

$1445(32.4)$

534 (12.0)

217 (4.9)

$1.99(1.43-2.77)$

$1.61(1.17-2.22)$

$1.35(0.97-1.87)$

$1.16(0.81-1.67)$

1.00

3033 (68.0)

1430 (32.0)

1.00

1.35 (1.23-1.47)

$54.8 \pm 9.4$

1.01 (1.01-1.02)

$163.5 \pm 7.8$

1.02 (1.01-1.02)

1481 (33.4)

$2155(48.6)$

798 (18.0)

1.00

$1.05(0.94-1.16)$

$1.12(0.98-1.28)$

2358 (53.2)

1813 (40.9)

259 (5.9)

1.00

$1.18(1.07-1.30)$

$1.06(0.87-1.30)$

483 (12.3)

1989 (50.7)

$917(23.4)$

396 (10.1)

140 (3.6)

1.00

$1.01(0.86-1.19)$

$1.15(0.96-1.37)$

$1.03(0.84-1.28)$

$1.30(0.99-1.72)$

691 (15.6)

1.00

$3738(84.4)$

$1.02(0.89-1.16)$

2308 (52.5)

2087 (47.4)

1.00

$1.12(1.02-1.23)$

4044 (93.2)

1.00

297 (6.8)

$1.31(1.11-1.55)$

2333 (53.0)

1.00

$2073(47.0)$

$2.10(1.91-2.31)$

$20(0.5)$

$4370(99.5)$

1.00

$0.65(0.24-1.74)$

$2455(55.2)$

1989 (44.8)

1.00

$0.79(0.72-0.87)$

1345 (33.1)

$1369(33.7)$

1352 (33.3)
1.00

1.49 (1.32-1.69)

1.83 (1.63-2.06)

${ }^{a}$ CI, confidence interval; MTC, motorcycle; kph, kilometers per hour.

${ }^{\mathrm{b}}$ Reference groups have a relative hazard equal to 1.00 .

${ }^{\mathrm{c}}$ Exchange of US\$ to NT\$ was approximately 1:26 at the time of the initial assessment. 
Table 3

Crude relative hazards of time-dependent characteristics for the occurrence of motorcycle crashes from the Anderson-Gill model analysis ${ }^{\mathrm{a}}$

\begin{tabular}{|c|c|c|}
\hline Characteristic $^{\mathrm{b}}$ & Frequency $(\%)$ at initial assessment & Relative hazard ${ }^{\mathfrak{c}}(95 \% \mathrm{CI})$ \\
\hline \multicolumn{3}{|l|}{ MTC riding, last month } \\
\hline Operator only & $1243(27.9)$ & 1.00 \\
\hline No riding & $801(17.9)$ & $0.42(0.35-0.50)$ \\
\hline Passenger only & $1140(17.9)$ & $0.54(0.47-0.61)$ \\
\hline Operator or passenger & $1279(28.7)$ & $0.93(0.84-1.04)$ \\
\hline \multicolumn{3}{|l|}{ Riding days, last 2 week } \\
\hline$\leq 1$ & $417(11.5)$ & 1.00 \\
\hline $2-3$ & $578(15.9)$ & $1.36(1.06-1.75)$ \\
\hline $4-6$ & $435(12.0)$ & $1.65(1.28-2.12)$ \\
\hline $7-9$ & $291(8.0)(17.2)$ & $1.84(1.41-2.40)$ \\
\hline $10-12$ & 626 & $1.96(1.55-2.47)$ \\
\hline $13-14$ & $1286(35.4)$ & $2.26(1.82-2.81)$ \\
\hline \multicolumn{3}{|c|}{ Riding time per riding day, last 2 week (min) } \\
\hline$\leq 10$ & $696(19.8)$ & 1.00 \\
\hline $11-20$ & $1331(37.8)$ & $1.29(1.07-1.55)$ \\
\hline $21-40$ & $839(23.8)$ & $1.52(1.29-0.79)$ \\
\hline $41-60$ & $174(4.9)$ & $1.73(1.46-2.05)$ \\
\hline $61-90$ & $321(9.1)$ & $2.08(1.67-2.61)$ \\
\hline$\geq 91$ & $161(4.6)$ & $2.19(1.84-2.60)$ \\
\hline \multicolumn{3}{|c|}{ Riding distance per riding day, last 2 week $(\mathrm{km})$} \\
\hline$\leq 1$ & $424(12.9)$ & 1.00 \\
\hline $2-5$ & $742(22.6)$ & $1.36(1.09-1.71)$ \\
\hline $6-10$ & $520(15.9)$ & $1.54(1.22-1.95)$ \\
\hline $11-20$ & $541(16.5)$ & $1.77(1.41-2.22)$ \\
\hline $21-50$ & $758(23.1)$ & $2.11(1.70-2.62)$ \\
\hline$\geq 51$ & $291(8.9)$ & $2.35(1.82-3.04)$ \\
\hline Past MTC crashes in study period & - & $1.55(1.48-1.62)$ \\
\hline \multicolumn{3}{|l|}{ Experience among operators (year) ${ }^{\mathrm{d}}$} \\
\hline$\leq 1$ & $1162(47.4)$ & 1.00 \\
\hline 1 & $468(19.1)$ & $0.91(0.79-1.04)$ \\
\hline 2 & $250(10.2)$ & $0.70(0.59-0.83)$ \\
\hline 3 & $247(10.1)$ & $0.87(0.72-1.05)$ \\
\hline 4 & $138(5.6)$ & $0.78(0.63-0.97)$ \\
\hline$\geq 5$ & $185(7.6)$ & $0.69(0.57-0.84)$ \\
\hline \multicolumn{3}{|l|}{ MTC license among operators ${ }^{\mathrm{d}}$} \\
\hline No & $1440(57.1)$ & 1.00 \\
\hline Yes & $1082(42.9)$ & $0.95(0.85-1.06)$ \\
\hline \multicolumn{3}{|l|}{ Automobile license among operators ${ }^{\mathrm{d}}$} \\
\hline No & $296(11.7)$ & 1.00 \\
\hline Yes & $2226(88.3)$ & $0.73(0.63-0.86)$ \\
\hline \multicolumn{3}{|l|}{ Helmet ownership } \\
\hline None & 1188 (32.6) & 1.00 \\
\hline Partial coverage & $628(17.2)$ & $1.38(1.20-1.59)$ \\
\hline Full coverage & $1829(50.2)$ & $1.40(1.26-1.57)$ \\
\hline \multicolumn{3}{|l|}{ Helmet use days, last 2 week } \\
\hline 0 & $1970(54.3)$ & 1.00 \\
\hline $1-3$ & $586(16.2)$ & $1.20(1.05-1.38)$ \\
\hline $4-6$ & $314(8.7)$ & $1.13(0.94-1.37)$ \\
\hline $7-9$ & $190(5.2)$ & $1.59(1.32-1.91)$ \\
\hline $10-12$ & $332(9.2)$ & $1.47(1.26-1.70)$ \\
\hline $13-14$ & $235(6.5)$ & $1.66(1.42-1.95)$ \\
\hline \multicolumn{3}{|l|}{ Usual riding speed (kph) } \\
\hline$\leq 20$ & 59 (1.6) & 1.00 \\
\hline $21-40$ & $557(15.4)$ & $1.37(0.81-2.31)$ \\
\hline $41-60$ & $2111(58.2)$ & $1.48(0.89-2.47)$ \\
\hline $61-80$ & $791(21.8)$ & $1.87(1.11-3.12)$ \\
\hline$\geq 81$ & $109(3.0)$ & $1.68(0.94-3.01)$ \\
\hline
\end{tabular}


Table 3 (Continued)

\begin{tabular}{|c|c|c|}
\hline Characteristic $^{\mathrm{b}}$ & Frequency $(\%)$ at initial assessment & Relative hazard ${ }^{\mathfrak{c}}(95 \% \mathrm{CI})$ \\
\hline \multicolumn{3}{|l|}{ MTC belongs to } \\
\hline The student & $1642(45.3)$ & 1.00 \\
\hline Family & $788(21.7)$ & $0.71(0.63-0.80)$ \\
\hline Classmate & $1041(28.7)$ & $0.61(0.54-0.69)$ \\
\hline Other & $152(4.2)$ & $0.68(0.53-0.87)$ \\
\hline \multicolumn{3}{|c|}{ Alcohol use, last month } \\
\hline No & $2427(66.7)$ & 1.00 \\
\hline Yes & $1212(33.3)$ & $1.40(1.27-1.54)$ \\
\hline \multicolumn{3}{|c|}{ Traffic violations, last 6 month } \\
\hline No & $637(17.5)$ & 1.00 \\
\hline Yes & $2996(82.5)$ & $1.54(1.38-1.72)$ \\
\hline \multicolumn{3}{|l|}{ Make of MTC } \\
\hline Yamaha & $1161(32.3)$ & 1.00 \\
\hline Sanyang & $1004(27.9)$ & $0.88(0.78-0.99)$ \\
\hline Kymco & $1238(34.5)$ & $0.90(0.80-1.01)$ \\
\hline Suzuki & $92(2.6)$ & $0.88(0.65-1.19)$ \\
\hline Other & $98(2.7)$ & $0.66(0.47-0.93)$ \\
\hline \multicolumn{3}{|c|}{ Engine volume of MTC $\left(\mathrm{cm}^{3}\right)$} \\
\hline 50 & $1668(46.5)$ & 1.00 \\
\hline $70-90$ & $210(5.9)$ & $1.03(0.84-1.27)$ \\
\hline $100-110$ & $118(3.3)$ & $1.14(0.88-1.48)$ \\
\hline 125 & $1256(35.0)$ & $1.06(0.95-1.17)$ \\
\hline$\geq 135$ & $332(9.3)$ & $1.01(0.84-1.21)$ \\
\hline \multicolumn{3}{|l|}{ Model year of MTC } \\
\hline$\leq 1990$ & $173(7.1)$ & 1.00 \\
\hline 1990 & $243(10.0)$ & $0.88(0.64-1.21)$ \\
\hline 1991 & $189(7.8)$ & $1.08(0.79-1.48)$ \\
\hline 1992 & $431(17.7)$ & $0.96(0.72-1.26)$ \\
\hline 1993 & $619(25.5)$ & $0.95(0.73-1.24)$ \\
\hline 1994 & $732(30.1)$ & $1.15(0.89-1.48)$ \\
\hline 1995-1996 & $44(1.8)$ & $1.54(1.16-2.03)$ \\
\hline \multicolumn{3}{|c|}{ Frequently used road type } \\
\hline One-way/1 lane & $211(6.0)$ & 1.00 \\
\hline One-way/ $\geq 2$ lanes & $260(7.4)$ & $1.01(0.79-1.30)$ \\
\hline Two-way/2 lanes & $1637(46.5)$ & $0.94(0.76-1.16)$ \\
\hline Two-way/4 lanes & 1219 (34.6) & $1.08(0.88-1.33)$ \\
\hline Two-way/ $\geq 6$ lanes & $193(5.5)$ & $1.21(0.92-1.57)$ \\
\hline
\end{tabular}

${ }^{\mathrm{a}} \mathrm{CI}$, confidence interval; MTC, motorcycle; kph, kilometers per hour.

${ }^{\mathrm{b}}$ Data on time-dependent characteristics are from students who rode a motorcycle at least once in the month prior to the assessment except for characteristics which are marked.

${ }^{\mathrm{c}}$ Reference groups have a relative hazard equal to 1.00 .

${ }^{d}$ Data from operators only.

crashes prior to the study were $(1.61)^{2} \times 1.61 \times(0.73)^{2}=$ 2.22 and $(1.61)^{2}=2.59$, respectively.

In the multivariate analysis, the risk of a crash also decreased with increasing age. Compared with operators with less than 1 year of experience, passengers or students who did not ride during the last month had a $40 \%$ lower risk of a crash $(\mathrm{RH}=0.60)$. After controlling for age at the initial assessment, students with more operating experience had a lower risk of being involved in a crash; for example, those with more than 5 years of experience had a $26 \%$ less risk of a crash $(\mathrm{RH}=0.74)$ than those with less than 1 year of experience. Furthermore, operators with an automobile license were $18 \%$ less likely to be involved in a crash than those without $(\mathrm{RH}=0.82)$. Alcohol use increased the risk of a crash by $13 \%(\mathrm{RH}=1.13)$. Students in both the middle and highest tertiles of risk-taking level were more likely to be involved in a crash than those in the lowest tertile $\left(\mathrm{RH}_{\text {middle }}=1.23, \mathrm{RH}_{\text {highest }}=1.24\right)$. Furthermore, traffic violations were associated with a $12 \%$ increase in risk $(\mathrm{RH}=1.12)$. Increases of each additional riding day $(\mathrm{RH}=1.02)$ or each additional riding hour $(\mathrm{RH}=1.07)$ or each 10 additional kilometers $(\mathrm{RH}=1.02)$ were associated with an increased risk of a crash. After controlling for the three riding exposures, neither helmet ownership nor the number of days of helmet use was associated with risk of a crash. 
Table 4

Adjusted relative hazards for the occurrence of motorcycle crashes from the Anderson-Gill model analysis ${ }^{\mathrm{a}}$

\begin{tabular}{|c|c|c|c|}
\hline Characteristic & Relative hazard $^{\mathrm{b}}$ & $(95 \% \mathrm{CI})$ & $P$-value \\
\hline Residential area (Hualien/Taipei) & 1.67 & $(1.29-2.17)$ & 0.000 \\
\hline Interaction of area with calendar time $e^{c}$ & 0.94 & $(0.92-0.97)$ & 0.000 \\
\hline Number of past MTC crashes during the study period & 1.61 & $(1.48-1.75)$ & 0.000 \\
\hline MTC crashes prior to the study (yes/no) & 1.61 & $(1.42-1.81)$ & 0.000 \\
\hline Interaction of the two past crash histories & 0.73 & $(0.66-0.81)$ & 0.000 \\
\hline \multicolumn{4}{|l|}{ Age at initial assessment (year) } \\
\hline 17 & 2.10 & $(1.49-2.94)$ & 0.000 \\
\hline 18 & 1.67 & $(1.20-2.32)$ & 0.002 \\
\hline 19 & 1.31 & $(0.94-1.83)$ & 0.108 \\
\hline 20 & 1.14 & $(0.79-1.65)$ & 0.486 \\
\hline$\geq 21$ & 1.00 & - & - \\
\hline \multicolumn{4}{|l|}{ MTC riding, last month } \\
\hline Operator with $<1$ year of experience & 1.00 & & \\
\hline Passenger only/no riding & 0.60 & $(0.39-0.91)$ & 0.016 \\
\hline \multicolumn{4}{|l|}{ Experience among operators (year) } \\
\hline$\leq 1$ & 1.00 & - & - \\
\hline 1 & 0.89 & $(0.77-1.02)$ & 0.097 \\
\hline 2 & 0.73 & $(0.62-0.87)$ & 0.001 \\
\hline 3 & 0.91 & $(0.75-1.11)$ & 0.336 \\
\hline 4 & 0.83 & $(0.67-1.03)$ & 0.091 \\
\hline$\geq 5$ & 0.74 & $(0.61-0.91)$ & 0.004 \\
\hline Automobile license among operators (yes/no) & 0.82 & $(0.69-0.96)$ & 0.015 \\
\hline \multicolumn{4}{|l|}{ Risk-taking level } \\
\hline Lowest tertile & 1.00 & - & - \\
\hline Middle tertile & 1.23 & $(1.08-1.40)$ & 0.002 \\
\hline Highest tertile & 1.24 & $(1.08-1.42)$ & 0.002 \\
\hline Alcohol use, last month (yes/no) & 1.13 & $(1.02-1.25)$ & 0.022 \\
\hline Traffic violations, last 6 month (yes/no) & 1.12 & $(0.99-1.27)$ & 0.063 \\
\hline Riding days, last 2 week (day) ${ }^{\mathrm{d}}$ & 1.02 & $(1.01-1.03)$ & 0.001 \\
\hline Riding time per riding day, last 2 week $(\mathrm{h})^{\mathrm{d}}$ & 1.07 & $(1.02-1.12)$ & 0.010 \\
\hline Riding distance per riding day, last 2 week $(10 \mathrm{~km})^{\mathrm{d}}$ & 1.02 & $(1.01-1.04)$ & 0.001 \\
\hline
\end{tabular}

${ }^{\text {a }} \mathrm{CI}$, confidence interval; MTC, motorcycle.

${ }^{\mathrm{b}}$ Reference groups have a relative hazard equal to 1.00 .

$\mathrm{c}$ The unit of calendar time is presented as a month.

d Null hypothesis of a linear association was not rejected.

\section{Discussion}

This is the first prospective study to examine the risk factors for all kinds of motorcycle crashes, both injury producing and non-injury producing, among 17-23-year-old young adults. The results of this study may be particularly helpful for areas or countries where motorcycles are used for the primary principal transportation.

Several findings have not been reported in previous studies. First, although, compared with a rural area such as Hualien, an urban area such as Taipei which has a lower risk of severe motorcycle injuries such as head injuries and fatalities (Hung et al., 1992; Lee et al., 1992), there is a greater risk of motorcycle crashes here. Second, the present study provides the first empirical evidence confirming the positive relationship between risk-taking level and the risk of motorcycle crashes. The relationship was independent of age, experience, and riding exposure. However, students in the highest tertile of risk-taking level were not at an increased risk of crashing compared to those in the middle tertile. Third, although higher riding speeds, after controlling for other factors in this study, did not have an independent association with the risk of motorcycle crashes, they have been reported to be related to an increased risk of motorcycle injuries (Kraus et al., 1975a; Hurt et al., 1981). More research is needed to validate whether riding speeds are related not to the incidence but to the severity of motorcycle crashes. Finally, the risk of a crash among students riding lower-powered motorcycles was no lower than those riding higher-powered motorcycles.

Two implications from this study on existing motorcyclerelated regulations in Taiwan should be noted. First, of motorcycle riders of all age groups in this study, those operators aged 17 , which is 1 year younger than the minimum legal age for a motorcycle license in Taiwan, had the highest risk of being involved in a crash. This finding suggests that police need to strictly enforce the minimum legal age laws for motorcycle riding to reduce the frequency of crashes among 
younger unlicensed riders. Second, in Taiwan, a driving test is not required to obtain a license to operate a motorcycle with an engine volume of $50 \mathrm{~cm}^{3}$ or smaller which comprises a substantial proportion of vehicles used among Taiwan's motorcycle riders (e.g. $47 \%$ in this study), even though these motorcycles can achieve speeds of up to $90 \mathrm{~km}$ per hour. However, our findings suggest that driving tests should also be given to applicants for a license to operate a motorcycle with an engine volume of $50 \mathrm{~cm}^{3}$ or smaller, like those for a license to operate a motorcycle of larger engine volume, to eliminate those unqualified in exercising ordinary and reasonable control in the operation of a motorcycle.

The effect of the residential area on the occurrence of motorcycle crashes changing over the study period might have resulted result from excluding the fifth-year Taipei students from this study as well as the inclusion of fifth-year Hualien students until their graduation in May 1995, who had a lower risk of crashing as compared to other students in the study. However, the interaction of residential area with calendar time does not disappear when excluding the fifth-year Hualien students in the multivariate analysis. Another possible explanation is that the road environments in Taipei might have changed to the detriment of motorcycle rider safety during the study period. This inference is suggested by the finding that the risk of among students from the two Hualien schools compared to the risk for students from the Taipei school at each follow-up assessment decreased in a similar manner (respective $\mathrm{RH}$ values were 1.72, 1.15 , and 0.96 at the three follow-up assessments for one school, and 2.45, 1.17, and 0.92 for the other). Furthermore, the monthly crash rate among students in Taipei increased over the study period. The ongoing construction of the mass transit transportation system under or over principal arterial roads near the study school in Taipei during the study period may have contributed to the increased risk of crashes. Also, new traffic regulations introduced during the study period restricted some travel lanes on many arterial roads to bus use only. These regulations further reduced the driving space separating cars and motorcycles in congested Taipei traffic, especially during peak traffic conditions.

There are at least three limitations to this study. One limitation is that the total number of reported motorcycle crashes might not have been complete, particularly during the period of winter and summer vacations, when contact between representatives and their classmates was likely to be less frequent. Winter and summer vacations usually occur in January/February and July/August. However, the crash rates in these 4 months were no lower than those in the other 8 months. Of the total crashes, about $80 \%$ were reported by class representatives, $20 \%$ were from crash victims at the three follow-up assessment visits, and information on two fatal crashes was obtained from school records. Almost all of the crashes reported by victims did not involve an injury. Furthermore, the ratio of all crashes to crashes requiring hospitalization was 13.3 for illegal riders aged less than 18 years and 25.5 for those aged 18 or above. This reveals that non-injury crashes may have been underreported by illegal riders, and that the risk of a crash at age 17 may still have been underestimated. Second, students lost to follow-up are likely to differ from those remaining in the study. Without the students graduating from schools immediately after the first follow-up assessment, those who did not complete the three follow-up assessments (23.2\%) were more likely to have had a crash prior to the study. Therefore, the overall risk of motorcycle crashes in the study sample and effects of these identified risk factors on motorcycle crashes might have been underestimated. Third, because of limitations of our prospective study design, environmental factors were not comprehensively measured. Consequently, it is possible that the study results were confounded by some unmeasured environmental factors.

In conclusion, a high-risk group predisposed to being involved in a motorcycle crash, including both non-injury and injury-related crashes, can be identified by using selected risk factors for crash prevention among young riders. Additionally, wearing a helmet does not increase the risk of being involved in a motorcycle crash.

\section{Acknowledgement}

This work was supported by the grant NSC 84-2331-b016-092 from the National Scientific Council, Republic of China.

\section{References}

American Medical Association, 1977. Head protection for the cyclist: a medical inquiry. Chicago, IL.

Andersen, P.K., Gill, R.D., 1982. Cox's regression model for counting processes: a large sample study. Ann. Stat. 10, 1100-1120.

Bach, E.R., Wyman, E.T., 1986. Financial charges of hospitalized motorcyclists at the Massachusetts general hospital. J. Trauma. 26, 343-347.

Baker, S.P., Fisher, R.S., 1977. Alcohol and motorcycle fatalities. Am. J. Public Health 67 (3), 246-249.

Begg, D.J., Langley, J.D., Reeder, A.I., 1994. Motorcycle crashes in New Zealand resulting in death and hospitalization. Part I. Introduction, methods and overview, methods and overview. Accid. Anal. Prevent. 26, 157-164.

Blincose L.J., 1994. The Economic Cost of Motor Vehicle Crashes, 1994. Technical report. US Department of Transportation, National Highway Traffic Safety Administration, Washington, DC.

Braddock, M., Schwartz, R., et al., 1992. A population-based study of motorcycle injury and costs. Ann. Emerg. Med. 21, 273-278.

Bray, T., Szabo, R., Timmerman, L., et al., 1985. Cost of orthopedic injuries sustained in motorcycle accidents. JAMA 254 (17), 2452-2453.

Chao, S.H., Tan, K.Y., 1984. Motorcycle injuries: an epidemiologic study of emergency room patients in large hospital in Taipei city. J. Natl. Public Health Assoc. (ROC) 4, 26-32.

Cook, R.J., Lawless, J.F., 1997. Marginal analysis of recurrent events and a terminating event. Stat. Med. 16, 911-924.

Ding, S.L., Wang, J.D., Chen, K.T., 1993. Estimate of case fatality rate and incidence rate of traffic injury in Taiwan: analysis of 4329 victims at a medical center. J. Formosan Med. Assoc. 92, S76-S81. 
Fleming, T.R., Harrington, D.P., 1991. Counting Process and Survival Analysis. Wiley, New York.

Hung, J.S., Chiu, W.T., Tsai, R.S., et al., 1992. Epidemiologic study of head injuries in Hualien County, Taiwan. J. Formosan Med. Assoc. 90, 1227-1233.

Hurt H.H., Quellet J.V., Thom D.R., 1981. Motorcycle accident cause factors and identification of countermeasures. Technical report, Vol. 1. US Department of Transportation, National Highway Traffic Safety Administration, Washington, DC.

Kraus, J.F., Riggins, R.S., Franti, C.E., 1975a. Some epidemiologic features of motorcycle collision injuries. Part I. Introduction, methods and factors associated with incidence. Am. J. Epidemiol. 102 (1), 74-98.

Kraus, J.F., Riggins, R.S., Franti, C.E., 1975b. Some epidemiologic features of motorcycle collision injuries. Part II. Factors associated with severity of injuries. Am. J. Epidemiol. 102 (1), 99-109.

Krishnan, R., Smith, G., 1994. Motorcycle injuries in South-east Asia. World Health Forum 15, 186-187.
Lee, L.S., Shin, Y.H., Chiu, W.T., et al., 1992. Epidemiologic study of head injuries in Taipei City, Taiwan. Chin. Med. J. (ROC) 50, 219-225.

Lin M.R., Huang W.Z., Hwang H.F. et al., 2002. The influence of crash involvement on risk taking among urban and rural adolescents in Taiwan, in submission.

McClure, R.J., Douglas, R.M., 1996. The public health impact of minor injury. Accid. Anal. Prevent 28, 443-451.

Ministry of Communication and Transportation, 1993. Annual report of transportation and communication in 1992. Executive Yuan, Taipei, Republic of China.

Rutledge, R., Stutts, J., 1993. The association of helmet use with the outcome of motorcycle crash injury when controlling for crash/injury severity. Accid. Anal. Prevent 25, 347-353.

Wei, L.J., Glidden, D.V., 1997. An overview of statistical methods for multiple failure time data in clinical trials. Stat. Med. 16, 833-839.

Wu, S.I., Yang, G.Y., Chou, P., et al., 1992. Analysis of traffic injuries in Taiwan in relation to alcohol use and economic loss. Injury 22, 357-361. 